\title{
Dichotic Listening in Patients With Splenial and Nonsplenial Callosal Lesions
}

\author{
Stefan Pollmann \\ University of Leipzig and \\ Max-Planck-Institute of Cognitive Neuroscience \\ D. Yves von Cramon \\ University of Leipzig and \\ Max-Planck-Institute of Cognitive Neuroscience
}

\author{
Marianne Maertens \\ Max-Planck-Institute of Cognitive Neuroscience
}

Joeran Lepsien

Max-Planck-Institute of Cognitive Neuroscience

\author{
Kenneth Hugdahl \\ University of Bergen
}

\begin{abstract}
The authors found splenial lesions to be associated with left ear suppression in dichotic listening of consonant-vowel syllables. This was found in both a rapid presentation dichotic monitoring task and a standard dichotic listening task, ruling out attentional limitations in the processing of high stimulus loads as a confounding factor. Moreover, directed attention to the left ear did not improve left ear target detection in the patients, independent of callosal lesion location. The authors' data may indicate that auditory callosal fibers pass through the splenium more posterior than previously thought. However, further studies should investigate whether callosal fibers between primary and secondary auditory cortices, or between higher level multimodal cortices, are vital for the detection of left ear targets in dichotic listening.
\end{abstract}

Contrary to the visual system, which has a clear contralateral organization up to primary visual cortex, the auditory pathways have multiple subcortical commissures, beginning as early as the superior olivary complex. Thus, to provide lateralized auditory input to a hemisphere, researchers had to devise a special technique. The dichotic listening (DL) technique, invented by Broadbent (1954), was developed by Kimura (1961) into a tool for the study of hemispheric function in normal subjects and brain-lesioned patients. In dichotic listening, two similar (but not identical) sounds are presented, one to each ear. Numerous studies have shown that under dichotic listening conditions, lexical material, such as words or consonant-vowel (CV) syllables, is detected more easily in the right ear in subjects with a language-dominant left hemisphere (LH; for an overview, cf. Hugdahl, 1995). The classic explanation of this right ear advantage (REA) rests on the assumption that contralateral auditory input dominates over ipsilateral input in DL. Thus,

Stefan Pollmann and D. Yves von Cramon, Day Clinic of Cognitive Neurology, University of Leipzig, Leipzig, Germany, and Department of Neurology, Max-Planck-Institute of Cognitive Neuroscience, Leipzig, Germany; Marianne Maertens and Joeran Lepsien, Department of Neurology, Max-Planck-Institute of Cognitive Neuroscience; Kenneth Hugdahl, Department of Biological and Medical Psychology, University of Bergen, Bergen, Norway.

We thank R. Rübsamen, F. Biedermann, and P. Bungert for making the audiometry data available.

Correspondence concerning this article should be addressed to Stefan Pollmann, Day Clinic of Cognitive Neurology, University of Leipzig, Liebigstrasse 22a, D-04103 Leipzig, Germany. E-mail: pollmann@cns.mpg.de material presented to the right ear will have direct access to the contralateral left hemisphere and therefore will be more easily detected than left ear input, which will have to cross the corpus callosum to reach the language-dominant hemisphere (callosal relay). Support for the callosal relay model came from studies of callosotomy patients. Whereas in normal subjects the right ear advantage is only a gradual difference between right and left ear stimulus detection (or identification), the disadvantage of the left ear was much more pronounced in patients with complete callosotomy (Milner, Taylor, \& Sperry, 1968; Sparks \& Geschwind, 1968). This left ear suppression in commissurotomy patients was expected if the contralateral pathway was dominant in DL, so that auditory transfer to the ipsilateral hemisphere could only be accomplished via the corpus callosum.

While the experiments with complete callosotomy patients demonstrated that the subcortical commissures were insufficient for ipsilateral transfer of auditory lexical material, subsequent studies investigated which partial lesions of the corpus callosum would disrupt ipsilateral transfer in DL. An early study (Springer \& Gazzaniga, 1975) found that left ear suppression was absent in a patient who had only the anterior third of the corpus callosum and the anterior commissure sectioned. There was also no left ear suppression in another patient with a splenial section. Contrary, left ear performance was deficient in another commissurotomy patient for which it was noted that "visual tests ... suggest that parts of the splenium were left intact" (Springer \& Gazzaniga, 1975, p. 342). Springer and Gazzaniga (1975) concluded that "sections anterior to the splenium and posterior to the first one third of the corpus callosum" (p. 341) were critical for left ear suppression. Alexander and Warren 
(1988) reported a case with a discrete lesion in the most posterior part of the trunk of the corpus callosum that showed complete left ear suppression for high frequency consonant-vowel-consonant (CVC) words presented dichotically as well as left-sided extinction of click sounds.

More recently, Sugishita et al. (1995) investigated DL performance in 5 patients with circumscribed lesions of the corpus callosum. Sugishita et al. were the first to present midsaggital magnetic resonance images (MRI) to document lesion location and extent in their patients. They concluded that lesions of the posterior $20-25 \%$ of the curvature of the corpus callosum led to left ear suppression. This area comprises the splenium and sometimes the most posterior part of the trunk of the corpus callosum. Thus, the emphasis was moved from the trunk of the corpus callosum to the splenium as the structure needed for auditory transfer to the LH.

The first aim of the present study was to investigate whether the relationship between splenial lesions and left ear suppression in DL can be replicated in a larger patient sample. A replication of the association between splenial damage and left ear suppression is important because this association was unexpected on the basis of the callosal relay model. As outlined above, the callosal relay model assumes that auditory fibers of the corpus callosum relay stimulus information to the LH. However, it is questionable whether the human splenium contains auditory fibers. Anatomical studies in the rhesus monkey locate the auditory commissures in the posterior part of the callosal trunk, rather than the splenium (Cipolloni \& Pandya, 1985). The sparse data available on the human corpus callosum seem to agree with this location, although they allow no exact localization of the auditory callosal fibers (De Lacoste, Kirkpatrick, \& Ross, 1985). A second aim of our study was to investigate to what degree directed attention to the left ear leads to better left ear performance in patients with splenial or nonsplenial callosal lesions. Under the assumption that $\mathrm{CV}$ syllable identification is an LH process, improved left ear detection would suggest at least a residual auditory transfer to the $\mathrm{LH}$ because an attentional enhancement can only be observed if stimulus transfer to the LH is not completely blocked.

Thirdly, different stimulus load may have led to conflicting results in previous studies, as pointed out by Sugishita et al. (1995). Wale and Geffen (1986) reported left ear suppression in a patient with a lesion of the genu. In contrast to the majority of studies, which have found no left ear suppression after lesions of the anterior third of the corpus callosum, Wale and Geffen used a dichotic monitoring paradigm, that is, patients had to monitor a rapid stream of sounds for the occurrence of a target. This may pose higher attentional demands than the classic procedure in which a pair of stimuli is presented, followed by ample time for the patient's response. We therefore tested our patients with both paradigms in order to investigate the influence of stimulus load on DL performance.

\section{Method}

\section{Subjects}

Ten former patients of the Day Clinic of Cognitive Neurology of the University of Leipzig who had partial lesions of the corpus callosum and 11 voluntary controls participated in the study after giving their informed consent according to the Declaration of Helsinki. Patients and controls were matched for age, gender, educational level, and handedness. The patient group comprised 6 men and 4 women and the control group contained 7 men and 4 women. The age range was between 20 and 66 years in the patients and between 21 and 66 years in the control group with a mean of 45 years ( $S D=16.23$ for controls and 16.19 for patients) in both groups. Table 1 summarizes the clinical data. Handedness was assessed with a German adaptation of the Edinburgh Handedness Inventory (Oldfield, 1971), and mean laterality quotients of 80 and 82 were obtained for the patient and the control groups, respectively. All participants were native German speakers.

Table 1

Patient Description: Demographic Data, Etiology, and Lesion Location

\begin{tabular}{|c|c|c|c|c|c|c|}
\hline $\begin{array}{l}\text { Patient } \\
\text { no. }\end{array}$ & $\begin{array}{l}\text { Age } \\
\text { (years) }\end{array}$ & Gender & $\begin{array}{l}\text { Handedness } \\
\text { (laterality } \\
\text { quotient) }\end{array}$ & $\begin{array}{l}Z \text { scores for } \\
\text { hearing loss }\end{array}$ & Etiology & Lesion sites \\
\hline 104 & 63 & $\mathrm{~F}$ & 100 & - & CVD: lacunar infarcts & $\mathrm{CC}$, left striatum, pons \\
\hline 126 & 66 & M & 100 & $0-0-0 / 1-0-0$ & ICH (operatively evacuated) & $\mathrm{CC}$, right parieto-occipital white matter \\
\hline 142 & 41 & $\mathrm{~F}$ & -4 & $3-0-0 / 3-1-1$ & $\begin{array}{l}\text { Craniopharyngeoma } \\
\quad \text { (transcallosally resected) }\end{array}$ & $\begin{array}{l}\mathrm{CC} \text {, hypothalamus, fornical columns, } \\
\text { internal capsule }\end{array}$ \\
\hline 197 & 57 & $\mathrm{~F}$ & 100 & $0-0-0 / 0-0-0$ & AcoA aneurysma (ruptured) & $\mathrm{CC}$, left frontomedian white matter, pons \\
\hline 286 & 60 & M & 100 & $0-0-0 / 0-0-0$ & CVD: lacunar infarcts & $\mathrm{CC}$, pons \\
\hline 311 & 34 & M & 57 & $0-0-0 / 0-0-0$ & TBI & $\mathrm{CC}$ \\
\hline 339 & 52 & M & 83 & $0-0-1 / 0-0-1$ & SAH (unknown origin) & $\mathrm{CC}$ \\
\hline 422 & 37 & M & 71 & $0-0-5 / 0-0-4$ & TBI & $\mathrm{CC}$ \\
\hline 424 & 20 & M & 88 & $5-3-0 / 3-2-0$ & TBI & $\begin{array}{l}\text { CC, right superior frontal gyrus, right } \\
\text { thalamus, right upper midbrain } \\
\text { tegmentum }\end{array}$ \\
\hline 432 & 20 & $\mathrm{~F}$ & 100 & $3-1-2 / 3-1-2$ & AVM (parieto-occipital) & $\mathrm{CC}$, left posterior thalamus \\
\hline
\end{tabular}

Note. $Z$ scores for hearing loss are hearing thresholds for the left/right ears at $250 \mathrm{~Hz}, 1000 \mathrm{~Hz}$, and $4000 \mathrm{~Hz}$. Dash indicates that data were not available. $\mathrm{F}=$ female; $\mathrm{M}=$ male; $\mathrm{CVD}=$ cerebrovascular disease; $\mathrm{CC}=$ corpus callosum; $\mathrm{ICH}=$ intracerebral hemorrhage; $\mathrm{Aco} \mathrm{A}=$ anterior communicating artery; $\mathrm{TBI}=$ traumatic brain injury; $\mathrm{SAH}=$ subarachnoidal hemorrhage; $\mathrm{AVM}=$ arterio-venous malformation. 


\section{Auditory Testing}

Patients' audiograms were acquired for eight pure tone signals between $0.125 \mathrm{kHz}$ and $16.0 \mathrm{kHz}$ separated by octave steps. Patients were tested in a soundproof booth (Industrial Acoustics, New York, NY) using headphones (Beyer Dynamics DT 770 Pro, Heilbronn, Germany). The psychoacoustic test procedure was automatized using a psychoacoustic setup (Tucker-Davis-Technologies [TDT, System II], Gainesville, FL) and associated software (SigGen, PsychoSig, TDT, Gainesville, FL). Table 1 contains hearing thresholds at $250 \mathrm{~Hz}, 1000 \mathrm{~Hz}$, and $4000 \mathrm{~Hz}$ in $Z$ scores, indicating deviation from normal thresholds. Although some patients had a hearing loss, especially at low frequencies, selective hearing loss in one ear was not observed.

\section{Lesion Measurement}

High-resolution, whole-head 3D modified driven equilibrium fourier transform (Lee et al., 1995; Ugurbil et al., 1993) magnetic resonance scans were obtained in 128 sagittal slices, with $1.5 \mathrm{~mm}$ thickness and a data matrix of $256 \times 256$ voxels. Figure 1 shows midsaggital sections of the corpus callosum of each patient. In addition, we ran a $\mathrm{T}_{2}^{*}$-weighted protocol in order to visualize microbleeds in patients with traumatic brain injury (TBI; Fazekas et al., 1999; Roob et al., 1999). The cases reported here did not show $\mathrm{T}_{2}$-visible periventricular white matter damage.

To measure the length of the corpus callosum quantitatively, we adopted the rostrum-splenium procedure by Sugishita et al. (1995). In the midsaggital plane, a curve was constructed manually, by means of a segmentation software (Kruggel \& Lohmann, 1996), midway between the dorsal and the ventral aspects of the corpus callosum from the tip of the rostrum to the end of the splenium. The length of this segmented curve was defined as the total length of the corpus callosum. The anterior and posterior limits of the callosal lesions were marked on this curved line, and the extent of the lesion was calculated as a percentage of the total corpus callosum length (see Figure 2). Following Sugishita et al. (1995), we categorized lesions affecting the posterior $20 \%$ as splenial and lesions within the anterior $80 \%$ as nonsplenial.

\section{Stimuli}

Pairs of CV syllables were presented dichotically via earphones (Sennheiser HD600, Tullamore, Ireland). The experimental stimulus set consisted of six CV syllables: /BA/, /DA/, /GA/, /KA/, /PA/, and $/ T A /$. In the entire experiment, the syllable $/ T A /$ was specified as target because in preliminary testing it produced the most pronounced right ear advantage with a right to left detection ratio of $2 / 3$ to $1 / 3$, respectively. Syllable duration was equated as far as possible (range $=338-359 \mathrm{~ms}$ ). The stimuli were digitized (as stereo VOC files, 16 bit, $22 \mathrm{kHz}$ ) and presented from a PC equipped with a Creative Laboratories SoundBlaster ViBRA 16X PnP board. After digitization, the dichotic syllable pairs were temporally aligned for simultaneous onset in the left and right channels and with regard to initial and final energy release with the aid of a stereo channel editing sound editor (Cool Edit; Syntrillium Software, 1995). Stimulus presentation was controlled by an ASCII running script made functional through the software package Experimental Run Time System (Berisoft, 1999).

\section{Design}

The experiment involved a $2 \times 2 \times 3$ factorial repeatedmeasures design with the factors ear of target presentation (left or right) and task (nonforced monitoring, forced left monitoring, verbal report) and the between-subjects factor group (patients vs. controls). In the nonforced monitoring task, subjects had to detect a prespecified target in a continuous stream of dichotically presented CV pairs. The forced left monitoring condition differed from the nonforced monitoring condition in that subjects were required to focus attention to the left ear. The verbal report task differed from the monitoring tasks in that the subjects responded verbally and had as much time as they needed to give the required response. The dependent variable in all tasks was detection accuracy.

\section{Procedure}

A typical experimental session lasted about $75 \mathrm{~min}$. It started with a pretest to assure that the participants were able to discriminate the syllables and to familiarize them with the experimental situation. Here the participants were presented with one syllable at a time either monaurally or binaurally and had to indicate orally if the stimulus was perceived to be "left," "right," or "middle." No subject committed more than two errors in about 20 trials. The pretest was followed by the actual experiment in the following order: (a) nonforced dichotic monitoring, (b) dichotic listening with verbal report, and (c) forced left dichotic monitoring. The forced left task was always run last in order not to bias attention to the left in the other two tasks.

Trials in the two monitoring tasks proceeded as follows. Presentation of the $\mathrm{CV}$ syllable pair was followed by a silent intertrial interval until presentation of the next $\mathrm{CV}$ pair. Trial duration was $800 \mathrm{~ms}, 1,000 \mathrm{~ms}$, or 1,200 ms to render the next stimulus onset unpredictable. Button press response was recorded in a time window of $800 \mathrm{~ms}$ from stimulus onset. Subjects had the instruction to press the left button of a response box with the right index finger whenever they detected the target stimulus. It was explicitly pointed out that it did not matter if the target was heard in the left or right ear. In the forced left monitoring task, subjects were instructed to concentrate exclusively on their left ear and report targets only if they were heard in the left ear. Subjects responded with the index finger of their left hand to eliminate callosal relay demand caused by crossed stimulus-response arrangement.

The verbal report task required subjects to report aloud whether the target was present or not ("yes" or "no"). The experimenter (Marianne Maertens) recorded the response by a button press that also (after a delay of $800 \mathrm{~ms}$ ) started the next trial. Subjects were instructed that the experimenter would wait for their response.

In all three tasks, /TA/ targets were present in 90 of 125 trials of a block. Targets were presented in the left and right ear channels in equal numbers. Conditions were alternated in a randomized fashion. The monitoring tasks (nonforced and forced left) consisted of five blocks each. The verbal report task, owing to its increased time demand per trial, consisted of three blocks. Altogether, each subject performed $2 \times 625$ trials in the monitoring tasks and 375 trials in the verbal report task. Each task began with a training block, the responses of which were discarded. The training blocks consisted of 125 trials for the nonforced monitoring task and 40 trials each for the subsequent verbal report and left ear monitoring tasks. Blocks were separated by breaks, the duration of which (beyond a minimum of $20 \mathrm{~s}$ ) could be chosen by the subjects. Each block began by three repetitions of the binaurally presented target $/ T A /$, to remind subjects of its sound.

\section{Data Analysis}

The ratio of left and right ear target detection was computed as a laterality score (LS) according to the following formula: $L S=$ 


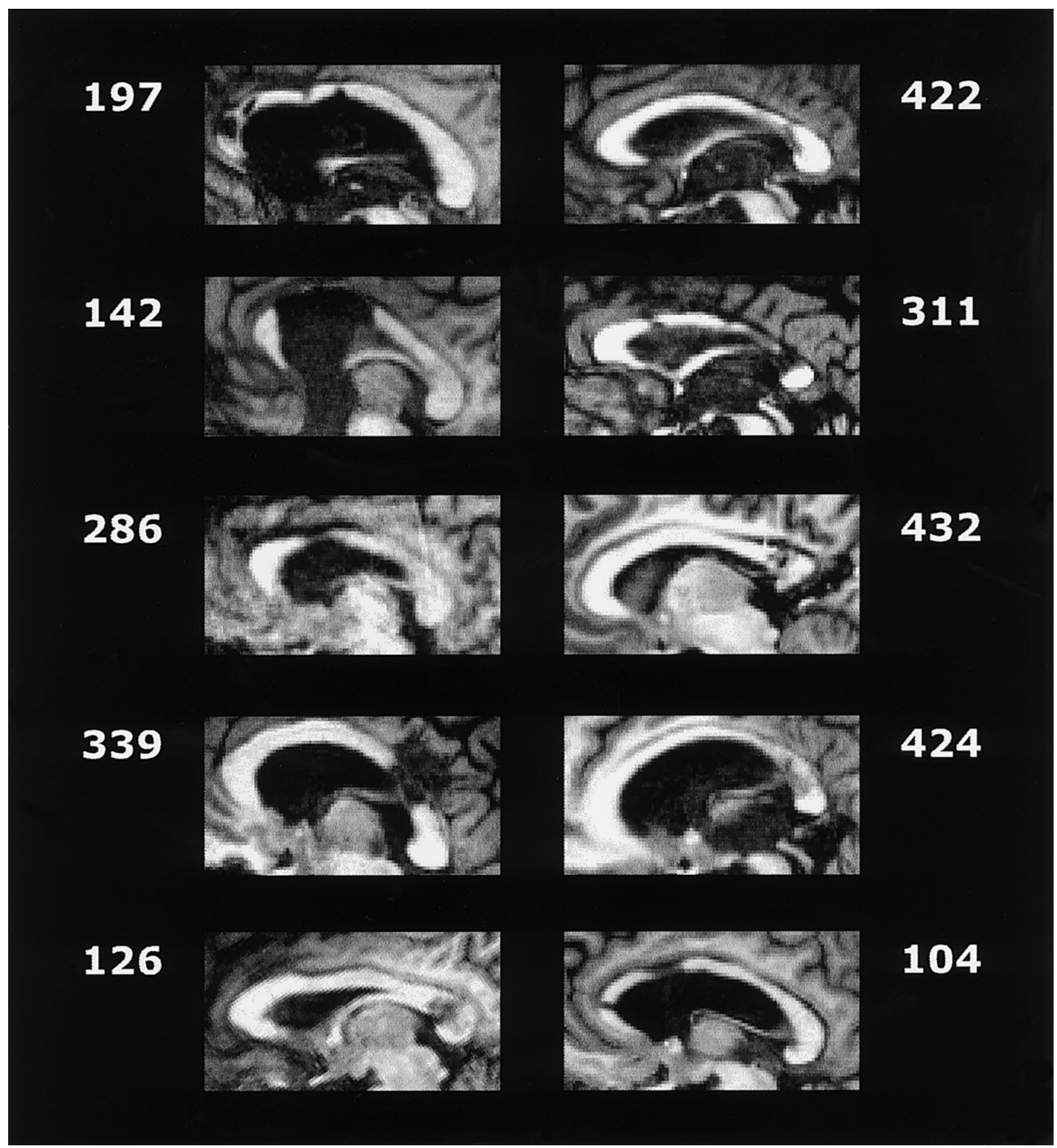

Figure 1. Midsaggital section of the corpus callosum in the 10 patients listed in Table 1. It should be noted that Patient 142 presents an additional lesion (not seen on the figure) in the left dorsolateral portion of the posterior callosal trunk anterior to the splenium. Numbers represent patient numbers.

$\left(D_{r}-D_{l}\right) /\left(D_{r}+D_{l}\right)$, where $D_{r}$ (or $\left.D_{l}\right)$ is the number of correct target detections in the right (or left) ear. Statistical data analyses were performed with nonparametric procedures. Positive scores indicate a relative advantage for the right ear stimulus (maximum $=1$ ). Negative scores indicate a relative advantage for the left ear stimulus (minimum $=-1$ ), whereas a score of zero indicates the absence of an ear advantage. The Wilcoxon Rank Sum Test was used for repeated-measures analysis, and the MannWhitney $U$ Test was used in the case of independent comparisons. Significance criterion was $\alpha=.05$.

\section{Results}

\section{Effects of Callosal Lesion}

As a whole, the patients did not differ from the normal control group in the number of target detections (in the left and right ear) in the nonforced monitoring task $(U=52$, $p=.833$; see Table 2). False-alarm rates did not differ between groups either (patients $=6 \%$, controls $=9 \%$; 

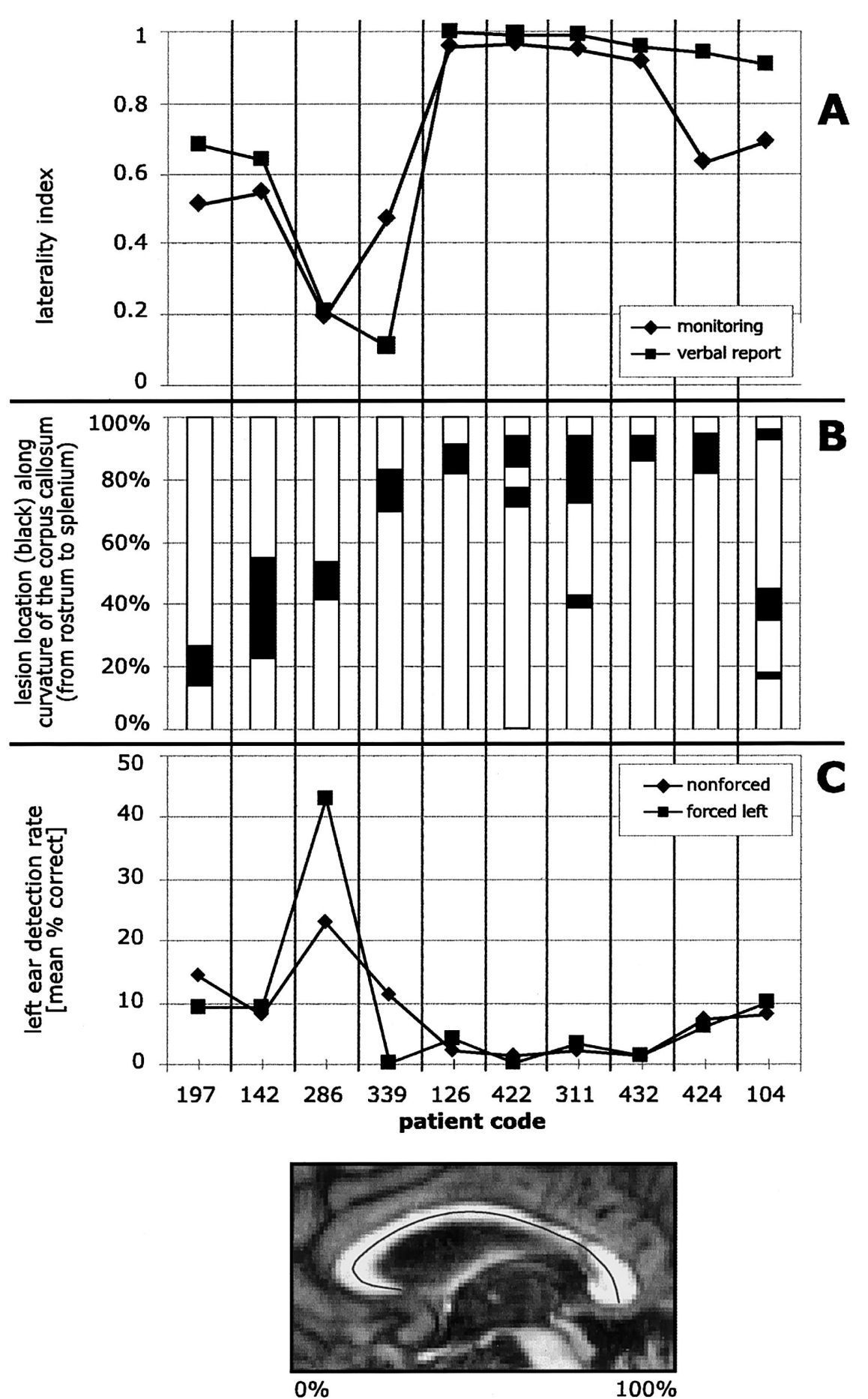

Figure 2. Individual patient performance in relation to lesion location. A: Laterality scores in the nonforced dichotic monitoring and verbal report tasks. B: Lesion location in percentage of the curvature of the corpus callosum from the tip of the rostrum $(0 \%)$ to the end of the splenium $(100 \%)$. $\mathrm{C}$ : Left ear detection rate in the nonforced and forced left dichotic monitoring tasks.

$U=39.5, p=.274)$, eliminating a response bias as a possible confounding factor. There was also no significant difference in the laterality scores between patients and controls, although laterality scores were higher (i.e., more right biased) in the patients $(U=27, p=.049)$.
In the verbal report task, the patient group had significantly higher laterality scores than the controls $(U=22$, $p=.020)$. However, there was no significant difference between the patients' laterality scores in the verbal report and the nonforced monitoring task $(Z=1.78, p=.075)$. 
Table 2

Behavioral Data for the Three Experiments

\begin{tabular}{|c|c|c|c|c|c|c|c|c|c|}
\hline \multirow{2}{*}{$\begin{array}{l}\text { Status and } \\
\text { Patient no. }\end{array}$} & \multicolumn{3}{|c|}{ Nonforced monitoring } & \multicolumn{3}{|c|}{ Forced-left monitoring } & \multicolumn{3}{|c|}{ Verbal report } \\
\hline & $f(R E)$ & $\mathrm{f}(\mathrm{LE})$ & $f(F A)$ & $\mathrm{f}(\mathrm{RE})$ & $\mathrm{f}(\mathrm{LE})$ & $f(F A)$ & $f(R E)$ & $\mathrm{f}(\mathrm{LE})$ & $\mathrm{f}(\mathrm{FA})$ \\
\hline \multicolumn{10}{|l|}{ Splenial } \\
\hline 104 & 0.41 & 0.08 & 0.05 & 0.12 & 0.10 & 0.01 & 0.67 & 0.03 & 0 \\
\hline 126 & 0.98 & 0.02 & 0.01 & 0.95 & 0.04 & 0.01 & 0.99 & 0 & 0.02 \\
\hline 311 & 0.89 & 0.02 & 0.02 & 0.32 & 0.03 & 0.02 & 0.95 & 0 & 0.01 \\
\hline 422 & 0.64 & 0.01 & 0.02 & 0.49 & 0 & 0 & 0.87 & 0 & 0 \\
\hline 424 & 0.29 & 0.07 & 0.10 & 0.28 & 0.06 & 0.10 & 0.95 & 0.03 & 0.01 \\
\hline 432 & 0.32 & 0.01 & 0.02 & 0.19 & 0.01 & 0.01 & 0.41 & 0.01 & 0 \\
\hline 339 & 0.31 & 0.11 & 0.14 & 0 & 0 & 0 & 0.25 & 0.20 & 0.20 \\
\hline$M$ & 0.55 & 0.05 & 0.05 & 0.33 & 0.03 & 0.02 & 0.73 & 0.04 & 0.03 \\
\hline$S D$ & 0.27 & 0.04 & 0.05 & 0.29 & 0.03 & 0.03 & 0.27 & 0.07 & 0.07 \\
\hline \multicolumn{10}{|l|}{ Nonsplenial } \\
\hline 142 & 0.29 & 0.08 & 0.03 & 0.27 & 0.09 & 0.07 & 0.17 & 0.04 & 0 \\
\hline 197 & 0.44 & 0.14 & 0.12 & 0.38 & 0.09 & 0.08 & 0.40 & 0.08 & 0.04 \\
\hline 286 & 0.33 & 0.23 & 0.05 & 0.07 & 0.43 & 0.04 & 0.68 & 0.44 & 0.05 \\
\hline$M$ & 0.35 & 0.15 & 0.06 & 0.24 & 0.21 & 0.06 & 0.42 & 0.19 & 0 \\
\hline$S D$ & 0.06 & 0.06 & 0.04 & 0.13 & 0.16 & 0.02 & 0.21 & 0.18 & 0.02 \\
\hline \multicolumn{10}{|l|}{ Control } \\
\hline 689 & 0.38 & 0.06 & 0.02 & 0.09 & 0.04 & 0.03 & 0.85 & 0.07 & 0.02 \\
\hline 1124 & 0.17 & 0.06 & 0.10 & 0.12 & 0.04 & 0.09 & 0.18 & 0.04 & 0.10 \\
\hline 1158 & 0.71 & 0.04 & 0.01 & 0.11 & 0.35 & 0 & 0.70 & 0.04 & 0.01 \\
\hline 1508 & 0.69 & 0.44 & 0.23 & 0.04 & 0.72 & 0.01 & 0.81 & 0.35 & 0.04 \\
\hline 1552 & 0.15 & 0.07 & 0.22 & 0.17 & 0.03 & 0.07 & 0.15 & 0.14 & 0.21 \\
\hline 1677 & 0.12 & 0.49 & 0.04 & 0.04 & 0.79 & 0.05 & 0.41 & 0.17 & 0.04 \\
\hline 1678 & 0.27 & 0.10 & 0.10 & 0.11 & 0.20 & 0.06 & 0.47 & 0.22 & 0.11 \\
\hline 2056 & 0.61 & 0.50 & 0.14 & 0.03 & 0.74 & 0 & 0.76 & 0.73 & 0.02 \\
\hline 2082 & 0.35 & 0.06 & 0.05 & 0.07 & 0.12 & 0.01 & 0.45 & 0.07 & 0.01 \\
\hline 2091 & 0.31 & 0.15 & 0.03 & 0.19 & 0.24 & 0.02 & 0.40 & 0.33 & 0.01 \\
\hline 2491 & 0.33 & 0.10 & 0.10 & 0.04 & 0.10 & 0.01 & 0.30 & 0.16 & 0.06 \\
\hline$M$ & 0.37 & 0.19 & 0.09 & 0.09 & 0.31 & 0.03 & 0.50 & 0.21 & 0.06 \\
\hline$S D$ & 0.20 & 0.18 & 0.07 & 0.05 & 0.29 & 0.03 & 0.24 & 0.19 & 0.06 \\
\hline
\end{tabular}

Note. $\mathrm{f}=$ relative frequencies; $\mathrm{RE}=$ right ear; $\mathrm{LE}=$ left ear; $\mathrm{FA}=$ false alarm.

Again, neither the overall detection rate $(U=44, p=.439)$ nor the false-alarm rate $(U=29.5, p=.070)$ differed between patients and controls in the verbal report task.

In the forced left monitoring task, the control group detected significantly more left ear targets, compared with the nonforced monitoring task $(Z=2.19, p=.028)$. In contrast, no such increase in the left ear detection rate was observed in the patients $(Z=0.36, p=.721)$. The increased detection rate of the control group was not due to a shift in response bias, as indicated by the equally low (3\%) falsealarm rates of patients and controls.

\section{Effects of Splenial and Nonsplenial Callosal Lesions}

Patients with splenial and nonsplenial callosal lesions did not differ in their overall target detection rate (all $U \mathrm{~s}>3$, $p>.087$ ) or false-alarm rate (all $U \mathrm{~s}>3, p>.086$ ) in any of the three tasks. In the laterality scores of the nonforced monitoring task, the patients with lesions anterior to the splenium $(n=3)$ did not differ from the control group $(U=16, p=.938)$. The splenial lesion group $(n=7)$, however, had significantly higher laterality scores than the controls $(U=11, p=.013)$. The same pattern was observed for the verbal report task (nonsplenial patient group vs. controls, $U=14, p=.697$; splenial group vs. controls, $U=8, p=.006$ ).
In both tasks, the splenial and the anterior lesion group did not differ significantly in their laterality scores, although the significance criterion was missed only slightly, especially in the nonforced monitoring task $(U=2, p=.053$; verbal report task, $U=3, p=.087)$. The individual laterality scores (see Figure 2) show that most patients with a lesion affecting the posterior $20 \%$ of the curvature from rostrum to splenium (the operational definition of splenium) had an almost total loss of target detection in the left ear. One patient of the splenial lesion group had lesions affecting only the anterior border zone of the splenium (Patient 339: posterior $17 \%$ of the corpus callosum intact). This patient had a normal REA. When Patient 339 was allocated to the subgroup with nonsplenial lesions $(n=4)$, the difference between the patient subgroups' laterality scores became significant for the nonforced monitoring as well as the verbal report task (both $U \mathrm{~s}=0, p=.011$ ).

The instruction to attend to left ear input did not increase the left ear detection rate in either the anterior or the splenial lesion subgroup, independent of the allocation of Patient 339 (with Patient 339 in splenial group, $Z=0.54, p=.593$, for the anterior, and $Z=0.09, p=$ .933 , for the splenial group, respectively, and with $\mathrm{Pa}-$ tient 339 in the anterior group, $Z=0, p=1$, and $Z=0.84, p=.4$, respectively). 


\section{Discussion}

We investigated the effects of partial lesions of the corpus callosum on dichotic listening. The main finding was that lesions affecting the splenium led to left ear suppression, whereas lesions of the corpus callosum anterior to the splenium did not affect laterality scores. This pattern of results was observed independent of stimulus load. Neither patients with splenial nor nonsplenial lesions benefited from focused attention to the left ear, contrary to normal controls.

Some of our patients had extracallosal lesions. However, these lesions were highly divergent, and no systematic relation to the behavioral data was observed. Another caveat is the etiology, which differed between patients; but again, there was no systematic relation between etiology and behavioral data. Thus, our data rather indicate that the observed effects are tied to the location of callosal lesion, independent of etiology. We went to great lengths to exclude magnetic-resonance-visible microlesions. However, we cannot exclude that patients may still have microlesions that are not visible with magnetic resonance methods.

\section{Specific Effects of Splenial Lesion}

Following Sugishita et al. (1995), we defined splenial lesions as lesions affecting the posterior $20 \%$ of the circumference from rostrum to splenium. Patients whose callosal lesions did not reach into the posterior $20 \%$ of the corpus callosum had, as a group, normal laterality scores. Contrary, patients with lesions affecting the posterior $20 \%$ of the corpus callosum showed left ear suppression. This was the case for the dichotic monitoring and verbal report tasks alike. Analysis of the individual laterality scores showed the clearest association between left ear suppression and splenial lesion in the verbal report task. This was surprising because the monitoring task, with its rapid succession of stimuli, was previously thought to be more demanding than the verbal report task, in which there was no time pressure, because the patient's response determined the duration of a trial (Sugishita et al., 1995). Thus, we found no evidence for the claim that patients with anterior callosal lesions may be susceptible to the higher stimulus load when dichotic stimuli in rapid succession had to be monitored. A potential confound could be that the verbal response, which is known to be LH dominant, prevented a direct response from the right hemisphere (RH). However, we have found no evidence for independent $\mathrm{RH}$ dichotic listening performance in the dichotic monitoring tasks with manual response either. Furthermore, because we are interested in the role of the corpus callosum in dichotic listening, a hypothetical capability of the RH to identify left ear targets would be beyond the scope of this article. We chose the verbal response because it is more natural than a forced-choice key press response. However, the data do not indicate that the manual response was problematic for our patients.

Whereas all patients with a nonsplenial lesion showed a normal REA, all but 1 patient with splenial lesions showed an almost complete left ear suppression. The 1 splenial patient (Patient 339) who did not show a left ear suppression had a lesion affecting the anterior border zone of the splenium, as defined in this article. This case indicates that the callosal fibers that are essential for left ear CV detection occupy an area that lies rather within the posterior $17 \%$ of the callosal circumference. This strengthens our interpretation that splenial lesions are associated with left ear suppression, because the posterior $20 \%$ of the corpus callosum, which we adopted as the operational definition of the splenium, often contains not only the splenium proper but also a part of the posterior trunk, as already pointed out by Sugishita et al. (1995). It should be noted that although we observed strong evidence for left ear suppression after purely splenial lesions (in Cases 126, 432, and 424, the lesion was restricted to the posterior $20 \%$ of the corpus callosum), the evidence for intact left ear target detection after posterior callosal body lesions hinges mainly on $\mathrm{Pa}$ tient 339. Replications of intact left ear detection in patients with posterior callosal body lesions would be desirable. The patients, both splenial and nonsplenial, did not differ in their detection rate (across left and right ear targets) or their false-alarm rate from the control group. This indicates that the observed left ear suppression was a pure laterality effect, which was not confounded by differential task difficulty or by a response bias.

\section{Focused Attention to the Left Ear}

The main deficit that was independent of lesion location was the patients' lack of benefit from focused attention to the left ear. Previous studies have shown that normal subjects benefit from the instruction to attend to a specific ear (Hugdahl, 1995). This benefit was replicated in the present study for the normal control group. The lack of a left ear attention benefit in the patients is in agreement with a previous dichotic listening study, in which the detection rate in the forced left condition was related to the intactness of the corpus callosum in patients with multiple sclerosis, whereas no such correlation was observed in the nonforced or forced right conditions (Reinvang, Bakke, Hugdahl, Karlsen, \& Sundet, 1994). Taken together, these data imply that the left hemisphere is vital for the detection of CV syllables under dichotic listening conditions. If the right hemisphere would be able to process this task independently, it is difficult to see why callosal lesions would eliminate the beneficial effect of directed attention to the left ear.

One possible explanation for the absence of a directed attention benefit after partial callosal lesions could be disruption of auditory transfer to the left hemisphere. If the auditory input does not reach the left hemisphere, attention has no input on which to work. However, although this may have been the case in individual patients, it seems safe to conclude that the diversity of the callosal lesion locations rules out disruption of auditory fibers as the only cause for the lacking attention effect in our patient group. It is likely that the corpus callosum is involved in interhemispheric transfer beyond initial transfer of auditory input. Callosal fibers transfer higher level information between the hemispheres, and disruption of interhemispheric processing can 
disrupt the processing of auditory stimuli at several stages from initial auditory transfer via stimulus identification to response preparatory processes.

In the forced left condition, patients and controls responded with their left hand, whereas responses in the nonforced condition were elicited with the right hand. This arrangement was chosen to provide uncrossed input to and output from the right hemisphere in the forced left condition. Since the data indicate that the left hemisphere was needed to carry out the task, it may be that the crossed response (from LH via $\mathrm{RH}$ motor cortex to left hand) led to a performance decrease in the forced left condition, which may have masked a gain from attending to the left ear. However, if such a trade-off between an attentional gain and a disruption of the crossed response may have happened, it should be kept in mind that there was a clear attend-left advantage in the control group and that this advantage was lost in presplenial and splenial patients alike, which makes disruption of interhemispheric motor transfer an unlikely explanation for the lack of the attend-left advantage.

\section{Functional Role of the Splenium}

To summarize, we found a clear association between splenial lesion and left ear suppression in dichotic listening. This association was independent of stimulus load. It was not ameliorated when the patients were asked to direct their attention to the left ear. However, a lack of directed attention was not a specific cause for the left ear suppression, because patients with nonsplenial lesions without left ear suppression showed the same lack of an attentional benefit.

This pattern of results is compatible with the classical callosal relay model of dichotic listening, which assumes that the left hemisphere is essential for the processing of lexical material and that the corpus callosum is needed for auditory transfer of left ear input to the left hemisphere because the ipsilateral input is suppressed in the auditory pathway with dichotic presentation. According to this model, we would have to conclude that the auditory commissures project through the splenium. This is in apparent contradiction with anatomical data on the location of auditory fibers in the corpus callosum. In the rhesus monkey, callosal fibers from primary auditory cortex have been found to occupy an area rostral to the splenium, in the posterior part of the callosal trunk (Cipolloni \& Pandya, 1985). Furthermore, the rostro-caudal position of the originating neurons in superior temporal gyrus was mirrored by an analogous rostro-caudal position of the respective fibers in the trunk of the corpus callosum. The same organizational principle, although on a much more coarse scale, was found in a degeneration study of the human corpus callosum. De Lacoste et al. (1985) found that lesions in the anterior temporal lobe led to degeneration in the anterior to middle third of the callosal trunk, while lesions in the posterior half of the temporal lobe led to degeneration in the posterior third of the trunk and the splenium. However, the latter lesion area included the whole temporo-parieto-occipital junction area, so that no exact information on the locus of the auditory fibers is available. Thus, if left ear suppression is due to disruption of interhemispheric auditory transfer, then the present data, along with the data of Sugishita et al. (1995), indicate that the auditory fibers in the human corpus callosum are located in the posterior $20 \%$ (and probably rather the posterior 17\%) of the curvature of the corpus callosum.

A recent positron emission tomography study of dichotic listening (Hugdahl, Brønnick, Law, Kyllingsbæk, \& Paulson, 1999) that used very similar stimuli as in the present study found bilateral superior temporal gyrus activation, including primary and secondary auditory cortices, when processing of CV syllables was compared with a tone discrimination baseline. If we assume that splenial lesions disrupted auditory transfer in the present study, the lesions may have affected commissures between primary or secondary auditory cortices. Thus, our data may indicate interspecies differences in the location of auditory callosal fibers. However, we cannot exclude that the balance between left and right ear stimulus detection may be disturbed at a level beyond auditory cortex. One critical question for further studies will be to test this alternative.

\section{References}

Alexander, M. P., \& Warren, R. L. (1988). Localization of callosal auditory pathways: A case study. Neurology, 38, 802-804.

Berisoft. (1999). Experimental Run Time System (Version 3.25) [Computer software]. Frankfurt am Main, Germany: Author.

Broadbent, D. E. (1954). The role of auditory localization in attention and memory span. Journal of Experimental Psychology, 47, 191-196.

Cipolloni, P. B., \& Pandya, D. N. (1985). Topography and trajectories of commissural fibers of the superior temporal region in the rhesus monkey. Experimental Brain Research, 57, 381-389.

De Lacoste, M. C., Kirkpatrick, J. B., \& Ross, E. D. (1985). Topography of the corpus callosum. Journal of Neuropathology and Experimental Neurology, 44, 578-591.

Fazekas, F., Kleinert, R., Roob, G., Kleinert, G., Kapeller, P., Schmidt, R., \& Hartung, H. P. (1999). Histopathologic analysis of foci of signal loss on gradient-echo T2*-weighted MR images in patients with spontaneous intracerebral hemorrhage: Evidence of microangiopathy-related microbleeds. American Journal of Neuroradiology, 20, 637-642.

Hugdahl, K. (1995). Dichotic listening: Probing temporal lobe functional integrity. In R. J. Davidson \& K. Hugdahl (Eds.), Brain asymmetry (pp. 123-156). Cambridge, MA: Bradford.

Hugdahl, K., Brønnick, K., Law, I., Kyllingsbæk, S., \& Paulson, O. B. (1999). Brain activation during dichotic presentations of consonant-vowel and musical instruments stimuli: A 15O-PET study. Neuropsychologia, 37, 431-440.

Kimura, D. (1961). Cerebral dominance and the perception of verbal stimuli. Canadian Journal of Psychology, 15, 166-171.

Kruggel, F., \& Lohmann, G. (1996). BRIAN (brain image analysis) - A toolkit for the analysis of multimodal brain datasets. In H. U. Lemke, K. Inamura, C. C. Jaffe, \& M. W. Vannier (Eds.), Computer assisted radiology: Proceedings of the International Symposium on Computer and Communication Systems for Image Guided Diagnosis and Therapy, Paris, June 1996 (pp. 323-328). Amsterdam: Elsevier.

Lee, J. -H., Garwood, M., Menon, R., Adriany, G., Andersen, P., Truwit, C. -L., \& Ugurbil, K. (1995). High contrast and fast three-dimensional magnetic resonance imaging at high fields. Magnetic Resonance in Medicine, 34, 308. 
Milner, B., Taylor, L., \& Sperry, R. W. (1968, July 12). Lateralized suppression of dichotically presented digits after commissural section in man. Science, 161, 184-186.

Oldfield, R. (1971). The assessment and analysis of handedness: The Edinburgh Inventory. Neuropsychologia, 9, 97-113.

Reinvang, I., Bakke, S. J., Hugdahl, K., Karlsen, N. R., \& Sundet, K. (1994). Dichotic listening performance in relation to callosal area on the MRI scan. Neuropsychology, 8, 445-450.

Roob, G., Schmidt, R., Kapeller, P., Lechner, A. Hartung, H. -P., \& Fazekas, F. (1999). MRI evidence of past cerebral microbleeds in a healthy elderly population. Neurology, 52, 991-994.

Sparks, R., \& Geschwind, N. (1968). Dichotic listening in man after section of neocortical commissures. Cortex, 4, 3-16.

Springer, S., \& Gazzaniga, M. S. (1975). Dichotic testing of partial and complete split brain subjects. Neuropsychologia, 13, 341346.
Sugishita, M., Otomo, K., Yamazaki, K., Shimizu, H., Yoshioka, M., \& Shinohara, A. (1995). Dichotic listening in patients with partial section of the corpus callosum. Brain, 118, 417-427.

Syntrillium Software. (1995). Cool Edit (Version 1.52) [Computer software]. Phoenix, AZ: Author.

Ugurbil, K., Garwood, M., Ellermann, J., Hendrich, K., Hinke, R., $\mathrm{Hu}, \mathrm{X}$., et al. (1993). Imaging at high magnetic fields: Initial experiences at 4T. Magnetic Resonance Quarterly, 9, 259.

Wale, J., \& Geffen, G. (1986). Hemispheric specialization and attention: Effects of complete and partial callosal section and hemispherectomy on dichotic monitoring. Neuropsychologia, 24, 483-496.

Received February 16, 2001

Revision received May 30, 2001

Accepted July 2, 2001

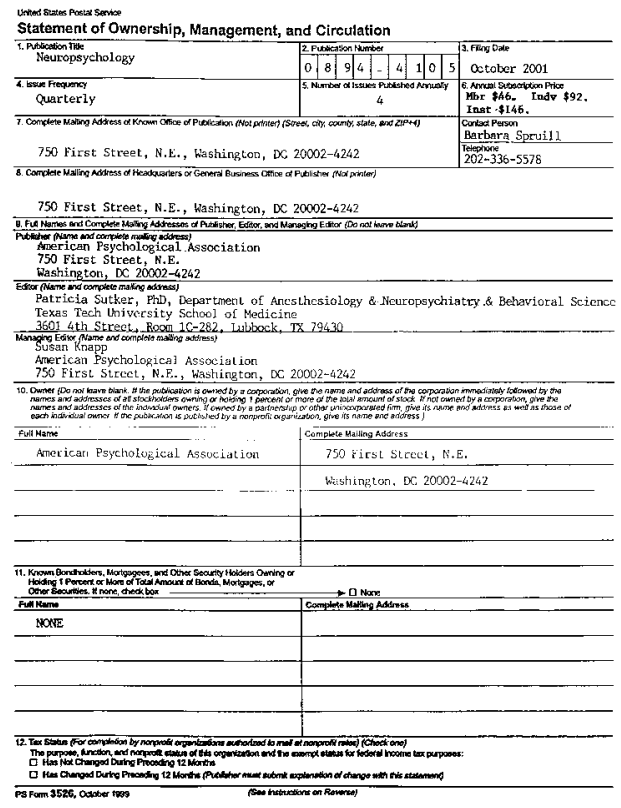

